\title{
Uso de bases de datos bibliográficas por investigadores biomédicos latinoamericanos hispanoparlantes: estudio transversal
}

\author{
Edgar Guillermo Ospina ${ }^{1}$, Ludovic Reveiz Herault ${ }^{1}$ \\ y Andrés Felipe Cardona ${ }^{2}$
}

Forma de citar Ospina EG, Reveiz Herault L, Cardona AF. Uso de bases de datos bibliográficas por investigadores biomédicos latinoamericanos hispanoparlantes: estudio transversal. Rev Panam Salud Publica. 2005:17(4): $230-6$.

RESUMEN Objetivo. Caracterizar cómo los profesionales biomédicos hispanoparlantes de América Latina acceden a y utilizan las bases de datos bibliográficas.

Métodos. A partir de una búsqueda en MEDLINE se identificaron 2515 artículos publicados entre agosto de 2002 y agosto de 2003 por autores de Argentina, Bolivia, Chile, Colombia, Costa Rica, Cuba, Ecuador, Guatemala, Honduras, México, Nicaragua, Panamá, Paraguay, Perú, Uruguay y Venezuela. La búsqueda se limitó a referencias de las ciencias básicas y clínicas y de la medicina social. Se envió una encuesta por correo electrónico a los autores que efectuaron su investigación y residian en alguno de los países estudiados. En la encuesta se exploraron el área de desempeño del investigador (ciencias básicas, clínicas o salud pública), el nivel de destreza en la utilización de las bases de datos, la frecuencia y el tipo de acceso a las bases de datos más empleadas, el impacto de la carencia de los textos completos en el momento de escribir un manuscrito y la vía mediante la cual los encuestados solían conseguir el texto completo de los artículos.

Resultados. Se enviaron en total 586 mensajes con la encuesta y se recibieron 185 respuestas (32\%). Las bases de datos más utilizadas para obtener información biomédica fueron MEDLINE (34,1\%), los motores de búsqueda generales (Google, Yahoo! y Alta Vista) (15,9\%), las revistas en línea $(9,8 \%)$, BIREME-LILACS (6,0\%), BioMedNet $(5,4 \%)$, las bases de datos del Centro para el Control y la Prevención de Enfermedades (CDC) de los Estados Unidos $(5,2 \%)$ y la Biblioteca Cochrane (4,9\%). De los encuestados, $64 \%$ indicaron tener habilidades medias o avanzadas en la utilización de MEDLINE. Sin embargo, $72 \%$ no utilizaban ni conocían los MeSH ("medical subject headings", términos estandarizados fijados por la Biblioteca Nacional de Medicina de los Estados Unidos para hacer búsquedas en bases de datos biomédicas). La frecuencia de las consultas a las bases de datos fue similar en todos los países estudiados, sin diferencias significativas en cuanto al tipo de acceso (formal, informal o libre) y el grado de habilidad. Del total, $87 \%$ reconocieron no haber incluido referencias importantes en artículos publicados por no disponer del texto completo y 56\% afirmaron haber citado artículos que no habían leído. Además, 7,6\% de los encuestados reconocieron haber consultado bases de datos de acceso restringido mediante claves prestadas o discos copiados. Más de dos tercios de los autores manifestaron que obtenían los textos completos de los artículos mediante fotocopia o directamente de los autores.

Clínica Reina Sofía, Santa Fe de Bogotá, Colombia. La correspondencia debe dirigirse a Edgar G. Ospina, Clínica Reina Sofía, Edif. Cons., Diagonal 127
A No. 31-48, Cons. 221, Bogotá, Colombia. Correo electrónico: ospina_edgar@yahoo.es
2 Instituto Nacional de Cancerología y Universidad Javeriana, Santa Fe de Bogotá, Colombia. 
Conclusiones. Es necesario entrenar a los investigadores latinoamericanos en la utilización de las bases de datos de uso más frecuente —especialmente MEDLINE-y mejorar su acceso a las fuentes bibliográficas biomédicas, como medidas esenciales para fomentar el desarrollo de la producción científica en la Región.

Palabras clave Bases de datos bibliográficas, acceso a la información, América Latina.

Después de la aparición de la escritura, la forma de transmisión, difusión y almacenamiento del saber científico permaneció estática hasta la aparición de la imprenta de Gutenberg, que generó un incremento vertiginoso en el proceso de distribución del conocimiento (1).

En la actualidad, Internet - cuyo uso se generalizó a partir de mediados de la década de 1980 - constituye una vía de acceso rápida y eficaz a las fuentes de almacenamiento y administración del conocimiento (2-4). El número de computadoras conectadas a Internet en América Latina se duplicó entre 1997 y 1999, cuando más de 5 millones de latinoamericanos $(1,5 \%$ de la población de este territorio) tenían acceso a esa red. Se estima que el número de computadoras conectadas en los países latinoamericanos aumentó en un 53\% anual entre 1999 y 2003 (5).

A pesar de la optimización de los procesos de comunicación, en la actualidad el conocimiento científico se sistematiza de manera caótica, y cada año se incorporan al acervo mundial aproximadamente 17000 libros y unos 30000 números de revistas biomédicas $(6,7)$. De igual forma, hay más de 1000 bases de datos relacionadas con las ciencias, lo que constituye un componente importante del modelo de comunicación científica actual. Esas bases de datos registran e indizan la información publicada y constituyen uno de los principales mecanismos para controlar y promover la publicación de resultados científicos $(8,9)$.

A partir de 1990 surgieron diversas iniciativas cuyo objetivo era lograr el acceso libre a los resultados de la investigación científica mediante bases de datos en Internet $(10,11)$. Sin embargo, en los países en vías de desarrollo, el acceso a los textos completos de la información biomédica sigue siendo limitado, a pesar de iniciativas como HINARI, BIREME y SciELO. Asimismo, no se han evaluado suficientemente la disponibilidad de la información contenida en las diferentes bases de datos y la destreza de los profesionales en su manipulación $(9,12,13)$.

MEDLINE es una base de datos de referencias bibliográficas que forma parte de PubMed y permite realizar búsquedas mediante palabras clave o encabezados de temas médicos conocidos como MeSH (del inglés medical subject headings). Estos términos forman un glosario controlado que se utiliza para garantizar que la indización de los artículos acerca de un tema específico sea coherente (14).

En los últimos tiempos se han empleado encuestas por Internet para investigar e impulsar la generación de hipótesis (15-17). Entre las limitaciones de las encuestas virtuales está la falta de representatividad de la muestra, por lo que su uso se limita por lo general a la evaluación de grupos específicos $(15,18-20)$.

El objetivo de este estudio fue caracterizar cómo los profesionales biomédicos hispanoparlantes de América Latina acceden a y utilizan las bases de datos bibliográficas.

\section{MATERIALES Y MÉTODOS}

Se realizó una búsqueda en MEDLINE mediante un filtro para seleccionar los artículos publicados entre agosto de 2002 y agosto de 2003 que contuvieran en los encabezados temáticos del MeSH o en la dirección de los autores el nombre de cualquiera de los siguientes 16 países: Argentina, Bolivia, Chile, Colombia, Costa Rica, Cuba, Ecuador, Guatemala, Honduras, México, Nicaragua, Panamá, Para- guay, Perú, Uruguay y Venezuela. La búsqueda se limitó a referencias de las ciencias básicas y clínicas (tanto humanas como no humanas) y la medicina social. La estrategia de búsqueda empleada tomó en cuenta los posibles sinónimos de los nombres de estos países y, especialmente, los errores por palabras homónimas.

La población de estudio abarcó a todos los autores latinoamericanos que publicaron en revistas indizadas en MEDLINE en el período estudiado. Se escogió MEDLINE por ser una de las bases de datos bibliográficos más extensa, porque indiza revistas que siguen procesos editoriales con arbitraje o revisión por pares y por ser de acceso libre.

La búsqueda identificó 2515 referencias, de las que se seleccionaron 586 autores cuya procedencia latinoamericana estaba claramente definida en la dirección postal y cuya dirección de correo electrónico indicara que vivían en alguno de los países estudiados. Se excluyeron los autores latinoamericanos que realizaron y publicaron sus estudios desde instituciones ubicadas fuera de América Latina o cuyas direcciones de correo electrónico indicaran que no residían en ninguno de los países estudiados, aunque la investigación se hubiera realizado en alguno de ellos. No hubo restricción en cuanto al tema o al tipo de documento encontrado en la búsqueda.

A pesar de que la investigación científica de Brasil es de las más cuantiosas de América Latina, los autores brasileños no se tomaron en cuenta en este estudio debido a problemas relacionados con el idioma.

Las direcciones de correo electrónico se organizaron por países en una base de datos digitada por duplicado y depurada para evitar que se registrara más de una vez al mismo autor. 
Se enviaron 586 mensajes de correo electrónico con una encuesta diseñada para el cuerpo del mensaje (sin archivos adjuntos). A los autores que no contestaron el mensaje en un plazo de dos semanas o cuyas encuestas llegaron incompletas o truncadas -entre ellos los casos en que se recibió una notificación de que el mensaje no le llegó al destinatario - se les envió un segundo mensaje con un asunto diferente en el encabezado con el fin de poder identificar a qué mensaje contestaba cada uno. El estudio finalizó cuatro semanas después de enviar el primer mensaje.

La encuesta contenía 10 preguntas estructuradas y una abierta. En la primera se preguntaba el área de desempeño del investigador (ciencias básicas, clínicas o salud pública); la segunda pregunta contenía una relación de 32 bases de datos a las que el encuestado podía añadir otras de su preferencia y luego seleccionar las cinco que más utilizaba. Las siguientes tres preguntas estaban dirigidas a determinar el nivel de destreza en la utilización de las bases de datos. También se preguntó el tipo de acceso a las bases con que contaba el investigador (licencia personal o institucional, libre acceso mediante Internet, o consulta informal o no autorizada), la frecuencia con la que usaba las bases de datos más empleadas, el impacto de no tener los textos completos en el momento de escribir un manuscrito y la vía por la cual el encuestado solía conseguir el texto completo de los artículos. Por último, los encuestados podían plasmar sus opiniones acerca del acceso libre de los investigadores latinoamericanos a las bases de datos.

La información de las encuestas respondidas fue digitada por duplicado en una base de datos y analizada mediante el paquete estadístico SPSS v. 11.0. Los mensajes que no encontraron destinatario se tabularon como rebotados y se excluyeron del análisis.

La tabulación de la frecuencia del uso de las cinco bases de datos más utilizadas por cada autor se realizó mediante un factor de ponderación, según su lugar en la preferencia (5 para la más utilizada y 1 para la menos empleada).
La suma de los resultados y su expresión porcentual permitieron conocer el orden de uso de cada base datos.

El índice de la frecuencia del uso de cada base de datos (IFU BDx) se calculó como la suma del número de veces que se prefirió cada una, ponderado según el orden de preferencia de su uso, mediante la siguiente ecuación:

$$
\begin{aligned}
\text { IFU BDx }= & \sum\left(F_{B D 1} \times 5\right)+\left(F_{B D 2} \times 4\right) \\
& +\left(F_{B D 3} \times 3\right)+\left(F_{B D 4} \times 2\right) \\
& +\left(F_{B D 5}\right)
\end{aligned}
$$

donde:

$B D x$ es cada una de las bases de datos biomédicas utilizadas por los encuestados.

$F_{B D 1 \ldots 5}$ es la frecuencia con que una base de datos fue elegida en los lugares $1 . . .5$ de preferencia por los investigadores encuestados.

Por su parte, para establecer la relación porcentual del uso de una base de datos con respecto a las otras se calculó el índice de la frecuencia de uso relativo (\% IFU BDx) mediante la ecuación:

$$
\% \text { IFU BDx }=\frac{I F U B D x}{\sum(I F U B D x)} \times 100
$$

Para el análisis de las variables cualitativas se utilizaron las pruebas de Pearson y de ji al cuadrado. Cuando el número de observaciones era menor de cinco se empleó la prueba exacta de Fisher. El nivel de significación escogido fue $P=0,05$.

\section{RESULTADOS}

Se enviaron en total 586 mensajes con la encuesta y se recibieron 185 respuestas $(31,6 \%)$. De ellas, $138(74,6 \%)$ correspondieron al primer mensaje y $47(25,4 \%)$ al segundo. De los mensajes enviados, $54(9,2 \%)$ rebotaron sin encontrar destinatario y $6(1,3 \%)$ de las respuestas no se tomaron en cuenta en el análisis porque llegaron truncadas (cortados por el servidor); del resto no se recibió respuesta.

Dos de los 16 países a los que se enviaron mensajes (México y Argentina) acumularon más de la mitad $(56,8 \%)$ de las publicaciones encontradas en MEDLINE con la estrategia de búsqueda empleada, seguidos por Chile, Colombia, Venezuela y Cuba (cuadro 1). De acuerdo con los mensajes enviados, se recibieron relativamente más respuestas de Colombia y México y menos de Guatemala y Ecuador. No se recibieron respuestas de Bolivia, Honduras o Paraguay.

Con respecto al área de desempeño de los 185 investigadores que respondieron la encuesta, $112(61 \%)$ se dedicaban principalmente a las ciencias

CUADRO 1. Referencias encontradas en la búsqueda y número de mensajes enviados y recibidos, según el país del autor

\begin{tabular}{lccccc}
\hline \multicolumn{1}{c}{ País } & $\begin{array}{c}\text { Referencias } \\
\text { encontradas }\end{array}$ & $\begin{array}{c}\text { Mensajes } \\
\text { enviados }\end{array}$ & $\%$ & $\begin{array}{c}\text { Mensajes } \\
\text { respondidos }\end{array}$ & $\%$ \\
\hline México & 872 & 206 & 23,6 & 81 & 39,3 \\
Argentina & 368 & 127 & 34,5 & 32 & 25,2 \\
Chile & 264 & 94 & 35,6 & 25 & 26,6 \\
Colombia & 182 & 46 & 25,3 & 19 & 41,3 \\
Venezuela & 121 & 33 & 27,3 & 8 & 24,2 \\
Cuba & 68 & 24 & 35,3 & 7 & 29,2 \\
Ecuador & 79 & 13 & 16,5 & 3 & 23,1 \\
Uruguay & 59 & 13 & 22,0 & 4 & 30,8 \\
Costa Rica & 90 & 8 & 8,9 & 2 & 25,0 \\
Perú & 177 & 8 & 4,5 & 2 & 25,0 \\
Guatemala & 67 & 6 & 9,0 & 1 & 14,3 \\
Panamá & 69 & 3 & 4.0 & 1 & 33,3 \\
Bolivia & 59 & 3 & 5,1 & 0 & 0 \\
Honduras & 13 & 1 & 7,7 & 0 & 0 \\
Paraguay & 26 & 1 & 3,8 & 0 & 0 \\
$\quad$ Total & 2514 & 586 & 23,3 & 185 & 31,6 \\
\hline
\end{tabular}


clínicas, $61(33 \%)$ a las ciencias básicas, $6(3 \%)$ a la medicina social y $6(3 \%)$ a las ciencias biológicas no humanas.

En cuanto a las bases de datos, la primera opción para realizar una búsqueda fue MEDLINE $(81,1 \%)$, con una frecuencia de uso diario de $36 \%$, semanal de $56 \%$ y mensual de $8 \%$. Los motores generales de búsqueda, como Alta Vista, Google y Yahoo!, fueron la segunda opción más empleada (14\%), con una frecuencia de uso diario de $73 \%$ y semanal de $27 \%$. Las bases de datos más utilizadas según el índice de frecuencia de uso relativo fueron MEDLINE $(34,1 \%)$ y los motores generales de búsqueda $(15,9 \%)$, seguidos por las revistas en línea $(9,8 \%)$, BIREME-LILACS $(6,0 \%)$ y BioMedNet $(5,4 \%)$ (cuadro 2$)$.

El nivel de destreza en la utilización de las bases de datos elegidas como primera opción fue avanzado según $94(52 \%)$ de los encuestados, mientras que $22(12 \%)$ manifestaron tener un dominio moderado y $68(36 \%)$ un dominio básico.

La mayoría de los usuarios de bases de datos encuestados respondieron que ingresaban a ellas semanalmente (cuadro 3). La frecuencia de consulta a las bases de datos fue similar en todos los países representados en la encuesta (cuadro 4), sin diferencias estadísticamente significativas en cuanto al tipo de acceso (formal, informal o libre) y nivel de habilidad.

De los 185 investigadores que respondieron la encuesta, $53(28,6 \%)$ manifestaron que utilizaban los términos $\mathrm{MeSH}$ en las búsquedas que realizaban, $49(26,5 \%)$ no los emplean habitualmente y $83(44,9 \%)$ desconocían su utilidad. Según un análisis independiente realizado entre los usuarios de MEDLINE para determinar la relación entre el nivel de destreza en su utilización y el uso de términos MeSH en las búsquedas, se encontró que de 159 autores que respondieron tener conocimientos medios o avanzados, $76 \%$ desconocían o no usaban los términos $\mathrm{MeSH}$; en cambio, entre los 24 que afirmaron tener conocimientos básicos para la utilización de MEDLINE, la mitad utilizaba las palabras clave en sus búsquedas $\left(c^{2}=7,15 ; \mathrm{df}=1 ; P=0,007\right)$.

CUADRO 2. Bases de datos según el orden en la preferencia de uso

\begin{tabular}{|c|c|c|c|c|c|c|c|}
\hline \multirow[b]{2}{*}{ Base de datos } & \multicolumn{5}{|c|}{ Preferencia de uso } & \multirow[b]{2}{*}{ IFUa } & \multirow[b]{2}{*}{$\% I^{b}$} \\
\hline & 1 & 2 & 3 & 4 & 5 & & \\
\hline Medline & 150 & 18 & 7 & 6 & 2 & 857 & 34,1 \\
\hline \multicolumn{8}{|l|}{ Motores generales de búsqueda } \\
\hline (Altavista, Google, Yahoo!, etc.) & 26 & 50 & 17 & 5 & 8 & 399 & 15,9 \\
\hline Revistas en línea & 3 & 26 & 27 & 16 & 14 & 246 & 9,8 \\
\hline Bireme-Lilacs & 2 & 20 & 13 & 10 & 3 & 152 & 6,0 \\
\hline BioMedNet & & 14 & 15 & 15 & 4 & 135 & 5,4 \\
\hline \multicolumn{8}{|l|}{ Centros para la Prevención y el } \\
\hline Control de Enfermedades (EUA) & & 6 & 21 & 16 & 12 & 131 & 5,2 \\
\hline Librería de la Colaboración Cochrane & & 8 & 21 & 13 & 3 & 124 & 4,9 \\
\hline Morbidity and Mortality Weekly Report & & & 8 & 10 & 16 & 60 & 2,4 \\
\hline Embase & & 10 & 4 & 2 & & 56 & 2,2 \\
\hline EBSCO & & 3 & 8 & 6 & 2 & 50 & 2,0 \\
\hline Proquest & & 6 & 4 & 2 & 5 & 45 & 1,8 \\
\hline Hinari & & 4 & 4 & & 5 & 33 & 1,3 \\
\hline Biosis & 2 & 3 & & 2 & 2 & 28 & 1,1 \\
\hline Emerging Infectious Diseases & & & & 13 & 2 & 28 & 1,1 \\
\hline MDConsult & & 3 & & 6 & & 24 & 1,0 \\
\hline OVID, Sciendirect & & 3 & 2 & 3 & & 24 & 1,0 \\
\hline WHOSIS & & 2 & 2 & & 3 & 17 & 0,7 \\
\hline HealthGate & & 2 & & & 6 & 14 & 0,6 \\
\hline Scielo & & 2 & 2 & & & 14 & 0,6 \\
\hline [Clau] Science Direct & 2 & & & & 2 & 12 & 0,5 \\
\hline Medscape & & & 2 & 2 & & 10 & 0,4 \\
\hline Abstracts of Reviews of Effectiveness & & & 2 & 1 & & 8 & 0,3 \\
\hline Oncoweb & & & & 4 & & 8 & 0,3 \\
\hline Scirus & & 2 & & & & 8 & 0,3 \\
\hline http://www.jormazabal.com & & & 2 & & & 6 & 0,2 \\
\hline CAB & & & 2 & & & 6 & 0,2 \\
\hline Highwire & & & & 2 & & 4 & 0,2 \\
\hline Health Technology Assessment & & & & 2 & & 4 & 0,2 \\
\hline UP TO DATE & & & & 2 & & 4 & 0,2 \\
\hline EuroSurveillance & & & & & 2 & 2 & 0,03 \\
\hline Canadian Medical Association & & & & & 2 & 2 & 0,03 \\
\hline Current Contents & & & & & 2 & 2 & 0,02 \\
\hline Synergy & & & & & 2 & 2 & 0,02 \\
\hline First Data Drug Database & & & & & & 0 & 0,0 \\
\hline WebFerret MetaSearch & & & & & & 0 & 0,0 \\
\hline BioMed Central & & & & & & 0 & 0,0 \\
\hline Infotrieve & & & & & & 0 & 0,0 \\
\hline Total & 185 & 182 & 163 & 138 & 97 & 2515 & 100 \\
\hline
\end{tabular}

a IFU: índice de frecuencia de uso.

b \%IFU: índice de frecuencia de uso relativo.

De los 140 usuarios frecuentes (al menos un acceso por semana) de MEDLINE, 30\% utilizaban palabras clave para optimizar sus búsquedas, mientras que de los 44 autores que las usaban con menos frecuencia, $16 \%$ reconocieron utilizar habitualmente los términos $\mathrm{MeSH}\left(\mathrm{c}^{2}=4,05 ; \mathrm{df}=1 ; P=\right.$ $0,04)$. Según el área de desempeño, el uso de términos MeSH fue más frecuente entre los que se dedicaban a la atención clínica $(30 \%)$ que entre los que trabajaban en ciencias básicas $\left(9,8 \% ; c^{2}=8,26 ; d f=1 ; P=0,004\right)$.
De los investigadores encuestados, $157(84,9 \%)$ utilizaban operadores booleanos para elaborar sus búsquedas, 19 $(10,3 \%)$ no los utilizaban y $9(4,8 \%)$ respondieron no conocerlos.

Los investigadores utilizaban las bases de datos de acceso gratuito cinco veces más que las de acceso pagado. Catorce autores $(7,6 \%)$ accedieron de manera informal (sin licencia, mediante claves de acceso prestadas o copias de discos) a alguna de las bases de datos comerciales, y EBSCO fue la más frecuentemente consultada de esta 
CUADRO 3. Frecuencia de acceso de los autores a las diferentes bases de datos de su preferencia

\begin{tabular}{lcr}
\hline \multicolumn{1}{c}{ Frecuencia } & $\begin{array}{c}\text { Número de } \\
\text { respuestas }\end{array}$ & $\%$ \\
\hline Diariamente & 123 & 17,9 \\
Semanalmente & 320 & 46,5 \\
Mensualmente & 186 & 27,0 \\
Esporádicamente & 59 & 8,6 \\
$\quad$ Total & 688 & \\
\hline
\end{tabular}

manera (cuatro investigadores), seguida por la Biblioteca Cochrane, alguna revista en línea, BIOSIS, Oncoweb y la plataforma Ovid.

Más de dos tercios de los autores encuestados manifestaron que obtenían los textos completos mediante fotocopia o directamente de los autores (cuadro 5).

En general, 61\% de los investigadores respondieron que al escribir artículos científicos para revistas biomédicas, en ocasiones habían tenido que prescindir de citar algunas referencias por no tener acceso a los textos completos, y $26 \%$ afirmaron haberlo hecho frecuentemente, mientras que solo $13 \%$ de los que respondieron la encuesta manifestaron que nunca habían tenido que hacerlo. Por otra parte, $47 \%$ de los encuestados dijeron haber publicado ocasionalmente estudios donde hacían referencia a artículos que no habían logrado conseguir en texto completo, mientras que $9 \%$ lo habían hecho frecuentemente y $44 \%$, nunca.

\section{DISCUSIÓN}

La revisión de la bibliografía científica es un componente importante de cualquier proyecto de investigación. La destreza para realizar búsquedas bibliográficas en bases de datos biomédicas es esencial para poder mejorar la comprensión de un tema específico, detectar brechas en el conocimiento, definir el objetivo de una investigación, elaborar estrategias metodológicas, e interpretar los resultados y establecer su relación con los de otros estudios.

Lamentablemente, las publicaciones seriadas latinoamericanas indizadas en MEDLINE representan menos de $2 \%$ del total de revistas de esa base de datos; de ellas, $70 \%$ provienen de tres países: Brasil (35\%), Argentina (18\%) y México $(17 \%)(9,21-23)$. Por otra parte, los profesionales latinoamericanos publican poco en revistas indizadas en MEDLINE. De 479731 referencias correspondientes al año 2000, solo 1,7\% eran de autores latinoamericanos o caribeños, y de ellas $46 \%$ eran de autores brasileños $(23,24)$. Algunos estudios han demostrado que la producción de América Latina se ha duplicado en los últimos 10 años; sin embargo, la distribución por países se mantiene sin grandes variaciones (23).

Según el presente estudio, los autores mexicanos y argentinos publicaron más de la mitad (57\%) de las publicaciones latinoamericanas encontradas en MEDLINE mediante la estrategia de búsqueda empleada (que no tomó en cuenta los artículos publicados por autores brasileños), seguidos por los

CUADRO 4. Accesos frecuentes ${ }^{\mathrm{a}}$ a las diferentes bases de datos, según el país del autor

\begin{tabular}{lcccc}
\hline \multicolumn{1}{c}{ País } & $\begin{array}{c}\text { Encuestados } \\
\text { que respondieron }\end{array}$ & $\begin{array}{c}\text { Accesos a } \\
\text { bases de datos }\end{array}$ & $\begin{array}{c}\text { Accesos } \\
\text { frecuentes }\end{array}$ & $\%$ \\
\hline México & 80 & 262 & 160 & 61,1 \\
Argentina & 32 & 116 & 66 & 56,9 \\
Chile & 24 & 195 & 165 & 84,6 \\
Colombia & 19 & 71 & 59 & 83,1 \\
Venezuela & 8 & 30 & 22 & 73,3 \\
Cuba & 7 & 30 & 24 & 80,0 \\
Uruguay & 4 & 12 & 10 & 83,3 \\
$\quad$ Total & 174 & 716 & 506 & 70,7 \\
\hline
\end{tabular}

${ }^{a}$ Accesos por usuarios que ingresaron a la base de datos al menos una vez por semana.
CUADRO 5. Forma de obtención de los textos completos

\begin{tabular}{lc}
\hline \multicolumn{1}{c}{ Forma empleada } & $\%$ \\
\hline Mediante fotocopia & 43,4 \\
Por solicitud al autor & 21,5 \\
Mediante pago & 15,1 \\
Suministrado por la base de datos & 15,1 \\
Suministrado por la industria & \\
$\quad$ farmacéutica & 3,7 \\
Acceso a la revistas en línea & 1,2 \\
\hline
\end{tabular}

chilenos, los colombianos, los venezolanos y los cubanos.

MEDLINE fue la base de datos más consultada y fue la primera opción de $81 \%$ de los autores encuestados, seguida de lejos por las otras bases de datos.

Para evaluar la destreza en la utilización de MEDLINE se han usado diversos indicadores, como el uso de límites en la elaboración de búsquedas, la combinación de términos con operadores booleanos y el empleo de las opciones basadas en términos $\mathrm{MeSH}$, artículos relacionados o búsquedas clínicas (clinical queries) disponibles en el sitio de PubMed. En un estudio sobre la búsqueda de fuentes por Internet realizada por médicos de familia, Cullen encontró que solo $10 \%$ de los usuarios de MEDLINE utilizaban habitualmente los términos MeSH. En el presente estudio, basado en una población con características diferentes, se encontró que $28,6 \%$ de los autores afirmaron conocerlos y usarlos (25).

A pesar de que $24 \%$ de los autores encuestados consideraron que su nivel de conocimientos y habilidades en la utilización de MEDLINE era moderado o avanzado, las respuestas recibidas demostraron que no dominaban las ventajas que ofrece PubMed para realizar las búsquedas y que no empleaban los términos $\mathrm{MeSH}$ en las búsquedas en MEDLINE, elementos indispensables para hacer buenas búsquedas avanzadas (25).

Uno de los problemas más importantes con el que se enfrentan los investigadores en América Latina es la dificultad para acceder a fuentes de información confiables y ordenadas que 
ofrezcan los textos completos. Esta carencia los obliga a recurrir en ocasiones a información parcial y sesgada y, como consecuencia, a generar información incompleta y con sesgos (13). La encuesta demostró que 88,5\% de los investigadores encuestados reconocieron haber prescindido de referencias al escribir sus manuscritos para publicación y más de la mitad $(50,7 \%)$ reconoció haber publicado estudios con referencias que no habían leído. Es importante resaltar que $21,4 \%$ de los encuestados afirmaron obtener el texto completo de los estudios necesarios para desarrollar su actividad científica mediante el contacto directo con los autores.

En una encuesta realizada por De Groote y colaboradores con personal sanitario de la ciudad de Chicago se demostró que $76 \%$ de los encuestados usaban la computadora diariamente para obtener información científica, $18 \%$ lo hacían semanalmente y $5 \%$ al menos una vez al mes. Solo $1 \%$ de los vinculados con las ciencias de la salud en los últimos cinco años indicó no haber utilizado la computadora o las bases de datos como medios para obtener textos de estudio (26). Los resultados obtenidos a partir de esta encuesta demuestran que la proporción de autores latinoamericanos hispanoparlantes que consultan las bases de datos diariamente es mucho menor $(17,9 \%)$, posiblemente por tener menos entrenamiento, equipos y redes, que en el caso de los profesionales esta- dounidenses. Además, existen diferencias notables entre los hábitos laborales y de actualización de unos y otros. Los latinoamericanos encuestados informaron que accedían cinco veces más a las bases de datos gratuitas y la mayoría de ellos $(64 \%)$ lo hacían al menos una vez por semana. La preferencia de los usuarios latinoamericanos por las bases de datos libres es similar a la de investigadores de otros lugares, como se observa en una investigación según la cual 79\% de los usuarios de PubMed lo preferían porque el acceso era libre (27).

Aunque indagar acerca de hábitos que se consideran vergonzosos puede generar sesgos $(17,18)$, en la muestra encuestada se encontró una proporción pequeña pero notable de autores que reconocieron haber accedido a la información biomédica mediante métodos informales, como claves prestadas y discos copiados. Esto puede ser un reflejo de los problemas que enfrentan los autores latinoamericanos para acceder a las bases de datos y a textos no gratuitos.

La tasa de respuesta a esta encuesta por correo electrónico (32\%) puede considerarse baja en comparación con las de otros métodos de encuesta, como las directas y las enviadas por postales o por fax, aunque estos métodos son más costosos y requieren de un mayor respaldo logístico y de tiempo (28). Si bien sensibilizar previamente a los encuestados mediante una comunicación personalizada genera mayores tasas de respuesta a las encuestas (29), limitaciones de presupuesto impidieron hacerlo en este estudio. No obstante, las tasas de respuesta a encuestas electrónicas superiores al $30 \%$ se consideran satisfactorias (30).

La presente investigación tiene algunas limitaciones. En primer lugar, la búsqueda en MEDLINE tuvo como objetivo reunir una muestra de los investigadores latinoamericanos con el mejor perfil de producción científica. No obstante, es posible que la exclusión de los autores de Brasil —uno de los mayores productores de literatura científica de la Región- pueda limitar el alcance de las conclusiones. En segundo lugar, es posible que los investigadores que añaden una dirección de correo electrónico a sus datos personales tengan mayor acceso a Internet y a las bases de datos que los investigadores que no lo hacen. Por último, los resultados alcanzados mediante encuestas no siempre pueden extrapolarse debido a posibles sesgos de selección que deben tenerse en cuenta al analizar los resultados $(15,30)$.

A pesar de las limitaciones mencionadas, los resultados de este estudio confirman la necesidad de entrenar a los investigadores latinoamericanos en la utilización de las bases de datos de uso más frecuente - especialmente de MEDLINE - y de ampliar su acceso a fuentes bibliográficas biomédicas, como medidas esenciales para promover el desarrollo de la producción científica en la Región.

\section{REFERENCIAS}

1. Eco U. From Internet to Gutenberg. The Italian Academy for Advanced Studies in America [serial online] 1995:996. Hallado en: http://www.hf.ntnu.no/anv/Finnbo/tekster/ Eco/Internet.htm. Acceso el 10 de enero de 2005.

2. Global Research. Global Internet statistics (by language) [sitio en Internet]. Hallado en: http://global-reach.biz/globstats/index. php3. Acceso el 10 de enero de 2005.

3. Biermann JS, Golladay GJ, Greenfield MLV. Evaluation of cancer information on the Internet. Cancer. 1999;86(3):381-90.

4. Glode LM. Cancer information on the world wide web. J Clin Oncol. 1997;15:29-33.
5. Jitanilab. Internet: proyeccciones en Latinoamérica, 2000. Hallado en: http://www.jitani. $\mathrm{com} / \mathrm{html} /$ jinews/jitani_pdfs/articulos_pdfs/ Internet.pdf. Acceso el 14 de enero de 2005.

6. Smith R. Where is the wisdom...? Br Med J. 1991;303:798-9.

7. Davidoff F, Haynes B, Sackett D, Smith R. Evidence based medicine. Br Med J. 1995;310: 1085-6.

8. Salvador JA, Angós JM. Directorio de bases de datos internacionales. Hallado en: http:// wzar.unizar.es/perso/bdl/Directorio.pdf. Acceso el 21 de enero de 2004.

9. Fernández MT, Gómez I, Sancho R, Morillo F, et al. Análisis de la producción científica en ciencias de la salud de los países de América Latina y el Caribe, período 1999-2000. Hallado en: http://www.ricyt.org/interior/ interior.asp?Nivel1=4\&Nivel2=1\&Idioma . Acceso el 14 de enero de 2005.

10. Movimiento por el acceso abierto a la literatura científica. Hallado en: http://www.info doctor.org/bibliotecapublicadelaciencia / index.php. Acceso el 10 de enero de 2005.

11. Delamothe T. Navigating across medicine's electronic landscape, stopping at places with Pub or Central in their names. Br Med J. 2001; 323:1120-2.

12. Pestaña A. El Medline como fuente de información bibliométrica de la producción es- 
pañola en biomedicina y ciencias médicas. Comparación con el Science Citation Index. Med Clin. 1997;109(13):506-11.

13. Veiga de Cabo J. Visibilidad de las revistas científicas e iniciativas para incrementar la difusión de las publicaciones españolas. Nutr Hosp. 2003;18:177-80. Hallado en: http:// www.grupoaulamedica.com/aula/nutricion/ n42003/EEditorial.pdf. Acceso el 10 de enero 2005.

14. University of Florida Health Science Center Libraries. Tutorial de PubMed. Miami: UFHSCL. Hallado en: http://www.sap.org.ar/Medline/tutorial/. Acceso el 10 de enero de 2005.

15. Eysenbach $G$, Wyatt J. Using the Internet for surveys and health research. J Med Internet Res. 2002;4(2):E13.

16. Eysenbach G, Diepgen TL. Labelling and filtering of medical information on the Internet. Meth Inf Med. 1999;38(2):80-8.

17. Dillman DA, Tortora RD, Bowker D. Principles for constructing web surveys 1998. Pullman, Washington: Washington State University; 1999. (SESRC Technical Report 98-50). Hallado en: http://survey.sesrc.wsu.edu/ dillman/papers/websurveyppr.pdf. Acceso el 10 de enero de 2005.

18. Schaefer DR, Dillmann DA. Development of a standard e-mail methodology: Results of an experiment. Revised version. Public Opinion Quarterly. 1998;62:378-97. Hallado en: http:// survey.sesrc.wsu.edu/dillman/papers /
E-Mailppr.pdf. 18 páginas. Acceso el 10 de enero de 2005

19. Dillman DA, Phelps G, Tortora R, Swift K, Kohrell J, Berck J. Response rate and measurement differences in mixed mode surveys using mail, telephone, interactive voice response and the Internet. Pullman, Washington: Washington State University; 2001. Hallado en: http:// survey.sesrc.wsu.edu/dillman/ papers/Mixed\%20Mode\%20ppr\%20_with\% 20Gallup_\%20POQ.pdf. Acceso el 10 de enero 2005.

20. Schleyer TK, Forrest JL. Methods for the design and administration of web-based surveys. J Am Med Inform Assoc. 2000;7(4): 416-25.

21. Red Iberoamericana de Indicadores de Ciencia y Tecnología (RICYT). Indicadores comparativos. Hallado en: http://www.ricyt.org/ interior $/$ interior.asp?Nivel1 $=4 \&$ Nivel2 $=1 \&$ Idioma. Acceso el 14 de enero 2005.

22. Cañedo R. Publicaciones seriadas latinoamericanas procesadas por la base de datos Medline. ACIMED. 2001;9(1):123-9. Hallado en: http://www.infomed.sld.cu/revistas/aci/vo 19_1_01/aci101001.pdf. Acceso el 10 de enero 2005.

23. Red Iberoamericana de Indicadores de Ciencia y Tecnología (RICYT). Publicaciones registradas en Medline. Hallado en: http:/ / www. ricyt.org/Indicadores/indicadores.htm. Acceso el 21 de enero 2003.
24. Rosselli DA. La investigación biomédica en Colombia: un análisis de Medline. Colombia Med. 1998;29:108-11.

25. Cullen RJ. In search of evidence: family practitioners use of the Internet for clinical information. J Med Libr Assoc. 2002;90(4):370-9.

26. De Groote SL, Dorsch JL Measuring use patterns of online journals and databases. J Med Libr Assoc. 2003;91(2):231-40.

27. Grefsheim S, Franklin J, Cunningham D. Biotechnology awareness study. Part 1: Where scientists get their information. Bull Med Libr Assoc. 1991;79(1):36-44.

28. Shawn RM, Iwaomoto M, Massouei MS, Yusuf HF, Stevenson JM, David F, et al. Comparison of e-mail, fax and postal surveys of pediatricians. Pediatrics. 2003;111(4):299-303.

29. Daley EM, McDermott RJ, McCormack Brown KR, Kittleson MJ. Conducting web-based survey research: a lesson in internet designs. Am J Health Behav. 2003;27(2):116-24.

30. Cook C, Heath F, Thompson RL. A metaanalysis of response rates in Web- or internetbased surveys. Educational and Psychological Measurement. 2000;60:821-36.

Manuscrito recibido el 26 de agosto de 2004. Aceptado para publicación, tras revisión, el 29 de diciembre de 2004.
ABSTRACT

\section{The use of bibliographic databases by Spanish- speaking Latin American biomedical researchers: a cross-sectional study}

Objective. To describe how Spanish-speaking biomedical professionals in Latin America access and utilize bibliographic databases.

Methods. Based on a MEDLINE search, 2515 articles published between August 2002 and August 2003 were identified that dealt with and/or had authors from 16 countries: Argentina, Bolivia, Chile, Colombia, Costa Rica, Cuba, Ecuador, Guatemala, Honduras, Mexico, Nicaragua, Panama, Paraguay, Peru, Uruguay, and Venezuela. The search was limited to references to basic science, clinical science, or social medicine. A survey was sent by e-mail to researchers who lived in 15 of the 16 countries (the exception being Nicaragua). The survey asked about the researcher's area of work (basic science, clinical science, or public health), the level of skill in using databases, the frequency and type of access to the databases most utilized, the impact from not having access to the full text of articles when preparing a manuscript, and how the respondent usually obtained the full-text version of articles.

Results. A total of 586 e-mail messages with the survey were sent out, and 185 responses were received $(32 \%)$. The databases most utilized to obtain biomedical information were MEDLINE (34.1\%), general search engines (Google, Yahoo!, and AltaVista) (15.9\%), on-line journals $(9.8 \%)$, BIREME-LILACS (6.0\%), BioMedNet (5.4\%), the databases of the Centers for Disease Control and Prevention of the United States of America (5.2\%), and the Cochrane Library (4.9\%). Of the respondents, $64 \%$ said they had average or advanced abilities in using MEDLINE. However, $71 \%$ of the respondents did not use or were not aware of the MEDLINE Medical Subject Headings (MeSH), a controlled vocabulary established by the National Library of Medicine of the United States of America for indexing articles. The frequency of accessing the databases was similar in all the countries studied, without significant differences in terms of the type of access (authorized access to commercial databases, unauthorized access to those databases, or access to databases available for free) or the level of abilities. Of the respondents, $87 \%$ said they had not included important references in the articles that they had published because they had not had access to the full text of those items, and $56 \%$ said they had cited articles that they had not read in full. In addition, $7.6 \%$ of the respondents admitted to unauthorized use of limited-access databases, such as through borrowed passwords or copied disks. More than two-thirds of the respondents said they obtained the full text of articles through photocopies or directly from the authors.

Conclusions. In order to encourage scientific output by Latin American researchers, more of them need to be trained in the use of the most frequently used databases, especially MEDLINE. Those researchers also need to have expanded access to the biomedical literature.

Key words Databases, bibliographic; access to information; Latin America. 